POS PROCEEDINGS

\title{
TMD quark distributions at small $\mathbf{x}^{*}$
}

\section{Martin Hentschinski ${ }^{\dagger}$}

Physics Department, Brookhaven National Laboratory, Upton, New York 11973, USA

E-mail: hentschabnl.gov

We discuss recent work on the transverse momentum dependent sea quark density at small $\mathrm{x}$ and its application to Drell-Yan production at forward rapidities

XXI International Workshop on Deep-Inelastic Scattering and Related Subject-DIS2013,

22-26 April 2013

Marseilles,France

${ }^{*}$ Work in collaboration with Francesco Hautmann and Hannes Jung

† Speaker. 


\section{Introduction}

Inclusive QCD scattering processes in the presence of a hard scale, are nowaday described with impressive accuracy through the framework of collinear factorization. Turing on the other hand to more exlcusive observables, such as the transverse momentum distribution of a hard final state, its power is becomes more and more limited. Considering for definitness the inclusive production of a $Z$-boson, one finds that collinear factorization provides a reliable estimate of the cross-section, even if one limits oneself to partonic cross-sections at leading order (LO) in the strong coupling $\alpha_{s}$. The transervers momentum distribution of the $Z$-boson is on the other hand within collinear factorization at LO obtained as delta function fixed at zero transverse momentum $p_{T}$. While the observed distribution can be recovered through the inclusion of higher order corrections, generating finite $p_{T}$ through the emissions of additional partons into the final state, the effectivness of the approach, present at the inclusive level, is lost.

A natural solution to this problem is to re-formulate factorization into partonic coefficent and parton distribution functions (pdfs) such that correct or at least well approximated kinematics is already re-covered at Born-level. In particular this requires to extend the framework of collinear factorization and to consider pdfs which carry information about the transverse momentum of partons inside the nucleon.

A possible starting point for the investigation of such distributions is provided by the high energy limit of QCD. For the above example of Z-production, this corresponds to the region of phase space where the hierachy

$$
\sqrt{s} \gg M_{Z} \gg \Lambda_{\mathrm{QCD}}
$$

holds, with $\sqrt{s}$ the center of mass energy of the scattering process. In this limit of phase space, perturbative QCD scattering amplitudes are known to factorize into convolutions of impact factors and a universal Green's function, which achieves the resummation of high energy logarithms $\ln s$, see [1]. For a recent application of high energy factorization at NLO to DIS see e.g. [2]. With the convolution integral of high energy factorized cross-sections given in terms $t$-channel transverse momenta, such a formalism allows formulate Transvers Momentum Dependent (TMD) factorization in the presence of the hierachy Eq. $(1.1)$, see [3, 4].

The current formulation of TMD pdfs, derived from high energy factorization, is mainly restricted to the quenched approximation, where only gluon and valence quark distributions are included $[5,6,7]$. This is at first natural, as the $t$-channel of QCD scattering amplitudes is at high center of mass energies dominated by gluon exchanges. That such a treatment is not entirely satisfactory, becomes immediatly apparent if one turns to the the description of quark induced final states which are sensitive to small $x$ resummation. This is for instance the case for the production of Z-bosons at LHC, both at forward and central rapidities. In such a case, dominant contributions from the small $x$ enhanced seaquark can be only included through $\mathscr{O}\left(\alpha_{s}\right)$ (forward) and $\mathscr{O}\left(\alpha_{s}^{2}\right)$ matrix elements, eliminating in this way one of the main initial motivations for the development of TMD factorization.

In this contribution we present a first step to address this issue by including sea quark contributions and examine forward Drell-Yan production. For further details we refer to [8]. 


\section{Definition of a TMD sea quark distribution and off-shell $q q^{*} \rightarrow Z$ coefficient}

The starting point for our definition of a TMD sea quark distribution is given by the the offshell TMD gluon-to-quark splitting function [4],

$$
P_{q g}\left(z, \frac{\boldsymbol{k}^{2}}{\boldsymbol{\Delta}^{2}}\right)=\left(\frac{\boldsymbol{\Delta}^{2}}{\Delta^{2}+z(1-z) \boldsymbol{k}^{2}}\right)^{2}\left[P_{q g}(z)+T_{R} 4 z^{2}(1-z)^{2} \frac{\boldsymbol{k}^{2}}{\boldsymbol{\Delta}^{2}}\right] .
$$

Here $\boldsymbol{\Delta}=\boldsymbol{q}-z \cdot \boldsymbol{k}$ with $\boldsymbol{k}$ and $\boldsymbol{q}$ transverse momenta of the off-shell gluon and quark respectively, while $z$ is the fraction of the 'minus' light cone momentum of the gluon which is carried on by the $t$ channel quark. $P_{q g}=T_{R}\left((1-z)^{2}+z^{2}\right)$ denotes the LO DGLAP gluon-to-quark splitting function. Although evaluated off-shell, the splitting probability is universal.

Unlike treatments which describe the gluon-to-quark splitting in terms of the LO DGLAP splitting function $P_{q g(z)}$, see e.g. [9], the TMD splitting function takes into account the entire tower of $\boldsymbol{k}^{2} / \boldsymbol{\Delta}^{2}$ corrections, which are suppressed in the strict collinear limit. The transverse momentum of the sea quark is in this way, after combination with the TMD gluon distribution, obtained as a consequence of subsequent branchings at small $x$, with no strong ordering in their transverse momenta.

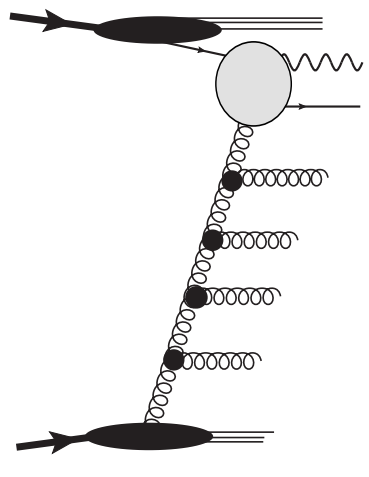

(a)

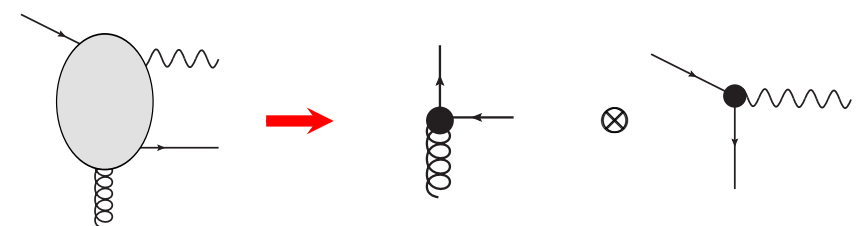

(b)

Figure 1: (a): If the vector boson is produced in the forward region, the sea quark density becomes sensitive to multiple small $x$ enhanced gluon emissions, leading to a $k_{T}$-dependent gluon density (b): Schematic factorization of the partonic $q g^{*} \rightarrow Z q$ process of a) into the $g^{*} \rightarrow q^{*}$ splitting and the $q q^{*} \rightarrow Z$ coefficient.

To relate the TMD splitting kernel to forward vector boson production, we analyze the flavor exchange process $g^{*} q \rightarrow Z q$, see Fig. 1. Within high-energy factorization, a factorized description of this process can be obtained within the "reggeized quark" calculus [10,11]. The latter extends the effective action formalism [12], currently explored at NLO [13], see also [14], to amplitudes with quark exchange in terms of effective degrees of freedom, the so-called reggeized quarks [15, 16]. The use of the effective vertices $[10,11]$ ensures gauge invariance of the coefficients relevant to perform the high-energy factorization $[3,4]$ for vector boson production, despite the off-shell parton.

Due to strong ordering of 'plus' and 'minus' light-cone momenta present in any high energy factorized amplitude, reggeized quark calculus leads for the $g^{*} q \rightarrow Z q$ process to a rather crude 
approximation to the $g^{*} \rightarrow q^{*}$ splitting function. Indeed, for Eq. (2.1) the strong ordering requirement corresponds to the limit $z \rightarrow 0$, which yields $P_{q g}=T_{R}$. It is however possible [8] to relax this kinematic restriction and to keep $z$ finite, while maintaining the gauge invariance properties of the original vertex. For the $g^{*} \rightarrow q^{*}$ splitting this yields then precisely the splitting function Eq. (2.1).

On the other hand, in the $q q^{*} \rightarrow Z$ coefficient the high energy limit sets the 'minus' component of the quark momentum to zero. It proves to be possible to relax the ordering prescription also in this case. It is thus interesting to investigate the effect of these kinematic corrections, which are subleading in the collinear and high energy limits. In [8] we express the off-shell coefficient for the $Z$-boson cross section as

$$
\hat{\sigma}_{q q^{*} \rightarrow Z}=\sqrt{2} G_{F} M_{Z}^{2}\left(V_{q}^{2}+A_{q}^{2}\right) \frac{\pi}{N_{c}} \delta\left(z x_{1} x_{2} s+T-M_{Z}^{2}\right) .
$$

Here the variable $T$ parametrizes the off-shellness of the $t$-channel quark. In the collinear limit $T \rightarrow$ 0 so that Eq. (2.2) agrees with the lowest order $q q \rightarrow Z$ coefficient. For the general off-shell case, $T$ interpolates between the squared transverse momentum of the off-shell quark, if strong minus momentum ordering is fulfilled, and modulus of the four-momentum transfer, if this condition is relaxed. Correspondingly, the $q g^{*} \rightarrow q Z$ cross section is expressed in terms of convolutions in transverse momentum and four momentum transfer [8] respectively. A numerical comparison of these two different realizations of off-shell factorization has been provided inr [8]. It reveals that for small $|\boldsymbol{\Delta}|$, the differences between $t$ and $k_{T}$-factorized expressions are numerically small, and both expressions are close to the full result; as $|\boldsymbol{\Delta}|$ increases, deviations due to the kinematic contributions by which the two expressions differ become non-negligible, and that the $t$-factorized expression gives a better approximation to the full result.

Future extensions of the above results will address the implementation of the result [17] into the Monte Carlo event generator CASCADE [18]. For a related study at partonic level which addresses $Z+$ jet production at high center of mass energies level see also [19]. Another direction of research addresses the extension to central $Z$ production, for which the $q_{T}$ factorized coefficient with two off-shell quark has been present in [20].

\section{Acknowledgments}

We acknowledge support from the U.S. Department of Energy under contract number DEAC02-98CH10886 and a BNL "Laboratory Directed Research and Development" grant (LDRD 12-034).

\section{References}

\section{References}

[1] V. S. Fadin, E. A. Kuraev and L. N. Lipatov, Phys. Lett. B 60 (1975) 50, I. I. Balitsky and L. N. Lipatov, Sov. J. Nucl. Phys. 28 (1978) 822 [Yad. Fiz. 28 (1978) 1597].

[2] M. Hentschinski, A. Sabio Vera and C. Salas, Phys. Rev. Lett. 110 (2013) 041601 [arXiv:1209.1353 [hep-ph]], Phys. Rev. D 87, 076005 (2013) [arXiv:1301.5283 [hep-ph]]. 
[3] S. Catani, M. Ciafaloni and F. Hautmann, Phys. Lett. B307 (1993) 147; Nucl. Phys. B366 (1991) 135; Phys. Lett. B242 (1990) 97.

[4] S. Catani and F. Hautmann, Nucl. Phys. B427 (1994) 475; Phys. Lett. B315 (1993) 157.

[5] M. Deak et al., arXiv:1012.6037 [hep-ph]; arXiv:1112.6354 [hep-ph]; arXiv:1112.6386 [hep-ph].

[6] E. Avsar, Int. J. Mod. Phys. Conf. Ser. 04 (2011) 74 [arXiv:1108.1181 [hep-ph]].

[7] F. Hautmann and H. Jung, JHEP 0810 (2008) 113; arXiv:0804.1746 [hep-ph].

[8] F. Hautmann, M. Hentschinski and H. Jung, Nucl. Phys. B 865 (2012) 54 [arXiv:1205.1759 [hep-ph]]. F. Hautmann, M. Hentschinski and H. Jung, in preparation.

[9] A. Gawron, J. Kwiecinski and W. Broniowski, Phys. Rev. D 68 (2003) 054001, M.A. Kimber, A.D. Martin and M.G. Ryskin, Phys. Rev. D63 (2001) 114027; Eur. Phys. J. C 12 (2000) 655, A.D. Martin, M.G. Ryskin and G. Watt, Phys. Rev. D70 (2004) 014012; Eur. Phys. J. C 31 (2003) 73, S. Höche, F. Krauss and T. Teubner, Eur. Phys. J. C 58 (2008) 17, A.D. Martin, M.G. Ryskin and G. Watt, Eur. Phys. J. C 66 (2010) 163.

[10] L. N. Lipatov and M. I. Vyazovsky, Nucl. Phys. B597 (2001) 399.

[11] A. V. Bogdan and V. S. Fadin, Nucl. Phys. B740 (2006) 36.

[12] L.N. Lipatov, Nucl. Phys. B452 (1995) 369; Phys. Rept. 286 (1997) 131.

[13] M. Hentschinski and A. Sabio Vera, Phys. Rev. D 85 (2012) 056006 [arXiv:1110.6741 [hep-ph]]; M. Hentschinski, Nucl. Phys. B 859 (2012) 129 [arXiv:1112.4509 [hep-ph]]; G. Chachamis, M. Hentschinski, J. D. Madrigal Martinez and A. Sabio Vera, Nucl. Phys. B 861 (2012) 133 [arXiv:1202.0649 [hep-ph]], Phys. Rev. D 87 (2013) 076009 [arXiv:1212.4992 [hep-ph]].

[14] A. van Hameren, P. Kotko and K. Kutak, JHEP 1301 (2013) 078 [arXiv:1211.0961 [hep-ph]], JHEP 1212 (2012) 029 [arXiv:1207.3332 [hep-ph]].

[15] V. S. Fadin and V.E. Sherman, Zh. Eksp. Teor. Fiz. 72 (1977) 1640;

[16] B.A. Kniehl, V. A. Saleev, A.V. Shipilova and E.V. Yatsenko, Phys. Rev. D 84 (2011) 074017; B.A. Kniehl, V. A. Saleev and A.V. Shipilova, Phys. Rev. D 79 (2009) 034007; V. A. Saleev, Phys. Rev. D 80 (2009) 114016; Phys. Rev. D 78 (2008) 114031. Pisma Zh. Eksp. Teor. Fiz. 23 (1976) 599.

[17] F. Hautmann, M. Hentschinski and H. Jung, arXiv:1205.6358 [hep-ph].

[18] H. Jung, S. Baranov, M. Deak, A. Grebenyuk, F. Hautmann, M. Hentschinski, A. Knutsson and M. Kramer et al., Eur. Phys. J. C 70 (2010) 1237 [arXiv:1008.0152 [hep-ph]].

[19] M. Hentschinski and C. Salas, arXiv:1301.1227 [hep-ph].

[20] M. A. Nefedov, N. N. Nikolaev and V. A. Saleev, Phys. Rev. D 87 (2013) 014022 [arXiv:1211.5539 [hep-ph]]. 